\title{
Role of the amygdala in the reinforcement omission effect
}

\author{
José Lino de Oliveira Bueno, Danielle Marcilio Judice-Daher, Tatiane Ferreira Tavares \\ Universidade de São Paulo, Ribeirão Preto, SP, Brazil
}

\begin{abstract}
The reinforcement omission effect (ROE) has been attributed to both motivational and attentional consequences of surprising reinforcement omission. Recent evidence suggests that the basolateral complex of the amygdala is involved in motivational components related to reinforcement value, whereas the central nucleus of the amygdala is involved in the processing of the attentional consequences of surprise. This study was designed to verify whether the mechanisms involved in the ROE depend on the integrity of either the basolateral amygdala complex or central nucleus of the amygdala. The ROE was evaluated in rats with lesions of either the central nucleus or basolateral complex of the amygdala and trained on a fixed-interval schedule procedure (Experiment 1) and fixed-interval with limited hold signaled schedule procedure (Experiment 2). The results of Experiment 1 showed that sham-operated rats and rats with lesions of either the central nucleus or basolateral area displayed the ROE. In contrast, in Experiment 2, subjects with lesions of the central nucleus or basolateral complex of the amygdala exhibited a smaller ROE compared with sham-operated subjects. Thus, the effects of selective lesions of amygdala subregions on the ROE in rats depended on the training procedure. Furthermore, the absence of differences between the lesioned groups in either experiment did not allow the dissociation of attentional or motivational components of the ROE with functions of specific areas of the amygdala. Thus, results did not show a functional double-dissociation between the central nucleus and basolateral area in the ROE. Keywords: reinforcement omission effect; basolateral amygdala; central nucleus of the amygdala; operant conditioning; rat.
\end{abstract}

Received 01 August 2012; received in revised form 04 December 2012; accepted 05 December 2012. Available online 28 December 2012.

\section{Introduction}

In animals that respond on intermittent schedules of reinforcement, response rates are often higher after reinforcement omission than after reinforcement. This reinforcement omission effect (ROE) has been attributed to both motivational and attentional consequences of the surprising omission of reinforcement. For example, Amsel \& Roussel (1952) reported that the introduction of partial reinforcement in the first goal of a double runway paradigm led to the greatest response on the second runway immediately after omission than after reinforcement delivery. This effect was explained by increments in the drive induced by primary frustration (Amsel \& Roussel, 1952; Amsel, 1992; Papini, 2003; Papini \& Dudley, 1997). However, ROE can be interpreted in terms of multiple processes that involve behavioral facilitation after nonreinforcement, and transient behavioral inhibition after reinforcement induced either demotivation or a resetting of the internal

José Lino de Oliveira Bueno, Danielle Marcilio Judice-Daher and Tatiane.Ferreira Tavares, Laboratório de Processos Associativos, Departamento de Psicologia, Faculdade de Filosofia, Ciências e Letras de Ribeirão Preto, Universidade de São Paulo - USP, Ribeirão Preto, SP, Brasil. Correspondence regarding this article should be directed to José Lino Oliveira Bueno, Faculdade de Filosofia, Ciências e Letras de Ribeirão Preto, Departamento de Psicologia, Universidade de São Paulo-USP, Avenida Bandeirantes, 3900, Ribeirão Preto, SP, Brazil. 14049-901. Phone: 55-16-6023697. Fax: 55-16-6335668. E-mail: jldobuen@usp.br clock (Seward, Pereboom, Butler, \& Jones, 1957; Staddon, 1974; Judice-Daher, Tavares, \& Bueno, 2011).

Some studies have suggested that lesions of the amygdaloid complex prevented the ROE (Henke, 1973, 1977; Henke \& Maxwell, 1973) showed that rats with amygdala lesions failed to alter running speed on a runway when reinforcement was omitted from the first goal box of a double runway. Henke (1973) also reported that rats with lesions of the amygdaloid complex and that were trained to respond on a fixed-interval (FI) 120-s schedule failed to increase response rates in the intervals that followed nonreinforcement. However, McDonough \& Manning (1979) found an opposite effect in which rats with lesions of the amygdaloid complex and that were trained to respond on a FI 60-s schedule were more responsive to occasional reinforcement omission than rats in the sham-operated group.

Recent evidence has shown that selective neurotoxic lesions of either the central nucleus of the amygdala (CeA) or basolateral amygdala (BLA) interfere with the ROE (Judice-Daher, Tavares, \& Bueno, 2012; Bueno, JudiceDaher, \& Tavares, 2012). The response rates of rats in both the BLA- and CeA-lesioned groups were higher compared with their respective sham-lesioned groups after reinforcement omission. Thus, lesioned rats were more sensitive to the omission effect. However, results showed that rats with CeA lesions - but not rats with BLA lesions - exhibited higher response rates following the omission of larger-magnitude reinforcement than the 
omission of smaller-magnitude reinforcement. Therefore, BLA lesions prevented differential performance after the omission of reinforcement of different magnitudes. This finding suggests a double-dissociation of the effects of the lesions. The BLA, but not CeA, may be involved in incentive processes relative to the omission of different reinforcement magnitudes. Therefore, the ROE may be modulated by brain circuitry that involves the amygdala.

Involvement of the amygdala in the modulation of the negative contrast effect has also been shown (Salinas, Parent, \& McGaugh, 1996; Salinas \& White, 1998). Although this effect is reflected by behavioral changes caused by incomplete reductions of reinforcement magnitude, similar processes are involved in both the ROE and negative contrast effect (Amsel, 1962). In a study by Salinas et al. (1996), large or selective neurotoxic amygdala lesions were induced after acquisition of a food-motivated runway approach task but prior to the reduction of reinforcement. The results showed that lesions of both the CeA and BLA and discrete lesions of the CeA did not interfere with the response to a reduction of reinforcement. However, discrete lesions of the BLA affected the negative contrast effect. Thus, the effects of a lesion of a specific amygdala nucleus will differ from lesions of other amygdala nuclei.

The view that blocking the ROE is attributable to amygdala damage is suggested by evidence that implicates the amygdala in responses that are correlated with emotional states (Salinas et al., 1996). The amygdala has long been implicated in several aspects of food-motivated associative learning including functions often characterized as attention, reinforcement, and representation. Each of these functions depends on the function of separate amygdala subsystems through their connections with other brain systems (Holland \& Gallagher, 1999). For example, the BLA is critical for the acquisition of positive incentive value induced by formerly neutral stimuli in Pavlovian and instrumental conditioning tasks and the ability of conditioned stimuli to gain access to the current motivational significance of unconditioned stimuli (Hatfield, Han, Conley, Gallagher, \& Holland, 1996; Holland \& Gallagher, 1999; Blundell, Hall, \& Killcross, 2001; Holland \& Gallagher, 2003; Balleine, Killcross, \& Dickinson, 2003). The CeA is involved in functions that may be described as attentional aspects: (1) modulation of orienting responses by associative learning and (2) enhancement of associability of particular events when expectancies about future events are violated (Holland \& Gallagher, 1993b, 1999; Everitt, Cardinal, Parkinson, \& Robbins, 2003).

The ROE has been attributed to both motivational and attentional processes. The present study sought to clarify whether the mechanisms involved in the ROE depend on the integrity of the BLA or CeA. Discrete bilateral lesions of either the CeA or BLA were induced after the acquisition of stable performance in pre-lesion training. Rats were trained on a FI schedule procedure (Experiment 1) and FI with limited hold signaled schedule procedure (Experiment 2). After surgical recovery, rats were subjected to sessions identical to those of the pre-lesion training, and the partial omission of reinforcement was subsequently introduced (postlesion testing). Performance of the rats with lesions of either the CeA or BLA and rats in the sham-operated group after reinforcement and nonreinforcement was compared.

\section{Experiment 1 \\ Methods}

Subjects. Thirty experimentally naive male Wistar rats, 90 days old at the beginning of the experiments and weighing 416-433 g were used in the study. Throughout the experiments, animals were singly housed in steel cages in the laboratory colony room on a 12-h light schedule (lights on 8:00 AM to 8:00 PM). Rats were kept on a water deprivation schedule at $85 \%$ of their $a d$ libitum body weight by limiting access to water. Food was freely available in their cages.

Apparatus. The behavioral training apparatus consisted of eight individual chambers $(20 \times 20 \times 23$ $\mathrm{cm})$ with aluminum front and back walls, clear acrylic sides and top, and a grid floor. A water cup was placed in the center of the left wall. A lever was located $7 \mathrm{~cm}$ above the floor and in the center of one of the walls. A 5 -W lamp located in the center of the ceiling constantly illuminated the chamber. A 7-W, 110-V lamp ("white light") was mounted $2 \mathrm{~cm}$ above the top of the chamber on the right side. This lamp was usually turned on and off according to the procedure. An interface (MRAElectronic Equipment, Ribeirao Preto, Brazil) connected the conditioning boxes to a computer that controlled the experiment and recorded the data. Each experimental chamber was kept inside a sound-proof wooden box (55 $\times 55 \times 55 \mathrm{~cm}$ ) with a $20 \times 15 \mathrm{~cm}$, transparent acrylic window. These sets were in a $6.0 \times 1.6 \times 3.0 \mathrm{~m}$ room. The interface and computer were in an adjacent room.

Pre-lesion training. In the first session, each rat was placed in the experimental chamber and trained to lever press for water. In the second session, which lasted $30 \mathrm{~min}$, animals were subjected to a continuous reinforcement frequency (CRF) schedule in which lever pressing at any moment was always followed by reinforcement. The next day, the rats were trained on a FI 60-s schedule. The first lever press after $60 \mathrm{~s}$ was always followed by water delivery (100\% reinforcement condition). During reinforcement, the white light in the experimental chamber was turned off for $3 \mathrm{~s}$ ("blackout"). Twenty training sessions, one session per day, were conducted throughout the experiment, Monday to Friday only. Each session lasted $50 \mathrm{~min}$. Rats were water-deprived for $\sim 23$ $\mathrm{h}$ before beginning each session.

Surgical procedures. After pre-lesion training, the animals underwent stereotaxic surgery according to the atlas of Paxinos \& Watson (2007). Surgery was performed under anesthesia induced by an i.p. injection of sodium pentobarbital (3\% Hypnol) diluted to $32.7 \%$. Each 
rat received $.3 \mathrm{ml}$ of anesthetic for each $100 \mathrm{~g}$ of body weight. Ten rats received bilateral lesions of BLA using the following stereotaxic coordinates: $2.8 \mathrm{~mm}$ posterior to bregma and $5.0 \mathrm{~mm}$ from the midline, with infusions 8.7 and $8.4 \mathrm{~mm}$ ventral to the skull surface. BLA lesions were made using $12.5 \mathrm{mg} / \mathrm{ml} N$-methyl-D-aspartate (NMDA; Sigma) in phosphate-buffered saline (PBS) solution infused with a $5-\mu 1$ Hamilton syringe $(.2 \mu 1$ at the deeper site and $.1 \mu 1$ at the shallower site). NMDA was used to make the BLA lesions because that agent tends to spare neurons in the neighboring CeA (Holland \& Gallagher, 2003). Ten rats received bilateral lesions of the CeA using the following stereotaxic coordinates: $2.3 \mathrm{~mm}$ posterior to bregma and $4.2 \mathrm{~mm}$ from the midline, with infusions at a depth of $7.9 \mathrm{~mm}$ from the skull surface. The CeA lesions were made using . $25 \mu \mathrm{l}$ of $10 \mathrm{mg} / \mathrm{ml}$ ibotenic acid (Sigma) in PBS solution. Ibotenic acid may induce the selective death of neurons and has been used to suppress enzymatic activity. In the present study, a permanent lesion was made because of the concentration/ dose used (Gallagher, Graham, \& Holland, 1990; Holland \& Gallagher, 1993a). Ten rats received the same surgical treatment (sham-operated group) with the exception that no solution was infused (Berlau \& McGaugh, 2003). All rats were allowed to recover from surgery for 2 weeks prior to post-lesion training.

Post-lesion training. After surgical recovery, rats were given three sessions of post-lesion training. These sessions were identical to the last sessions of the prelesion training (100\% reinforcement condition).

Post-lesion testing. After post-lesion training, three sessions of a fixed-interval/variable-time concurrent schedule (conc. FI-VT) were conducted during which reinforcement omission was introduced in $50 \%$ of the FI 60 -s schedule (partial reinforcement condition). In the reinforced trials, the first lever press after the FI produced water delivery followed by a "blackout" period. During nonreinforced trials, the first lever press after the FI produced only the "blackout." Reinforced and nonreinforced events were randomly distributed during the session using the criteria of up to three subsequent intervals with the same schedule.
The conc. FI-VT schedule procedure was used to equalize the number of reinforcements delivered per session, avoiding a specific effect of the amount of reinforcement on the subjects' behavior. Using this design, no performance alterations related to the reduction of reinforcements delivered would occur, only the local effect of reinforcement omission.

Histology. After the completion of the behavioral procedure, rats were deeply anesthetized with an i.p. injection of an anesthetic solution that contained sodium pentobarbital (3\% Hypnol) diluted to $32.7 \%$. Rats were then perfused intracardially with $400 \mathrm{ml}$ of a sulfide solution. The brains were removed and fixed in Carnoy solution and were then processed until final embedding in paraffin. Sections $(40 \mu \mathrm{m})$ were taken from each brain, and alternate sections were mounted on slides and stained with Cresyl violet. Slides were examined under a microscope, and neural structures were identified according to the stereotaxic atlas of Paxinos \& Watson (2007). The lesions were detected as the absence of visible neurons, often associated with a degree of tissue collapse and gliosis (visible as the presence of smaller, densely stained cells).

Data analysis. For statistical analysis, the average percentage of responses during the FI 60-s was grouped in 15-s periods. These data were analyzed using twoway analysis of variance (ANOVA) with group as an inter-group factor (BLA, CeA, and sham-operated groups) and period as an intra-group factor $(0-15-\mathrm{s}$, 16-30-s, 31-45-s, and 46-60-s periods). ANOVA was followed by the Newman-Keuls post hoc test; $p \leq .05$ was considered statistically significant.

\section{Results}

Histological results. Reliability criteria were established for the analysis of the lesions based on the atlas of Paxinos \& Watson (2007) by qualitatively comparing the borders, extent, and homogeneity of both intact and damaged structures. In all cases, the lesion sites were marked by heavy gliosis. At higher magnification, intact neurons were clearly visible at the borders of the lesions (Figure 1).

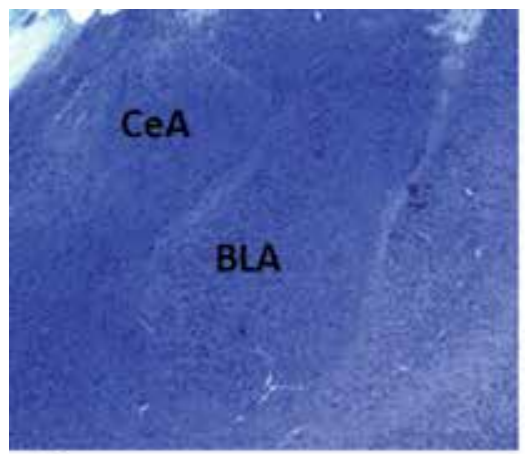

(A)

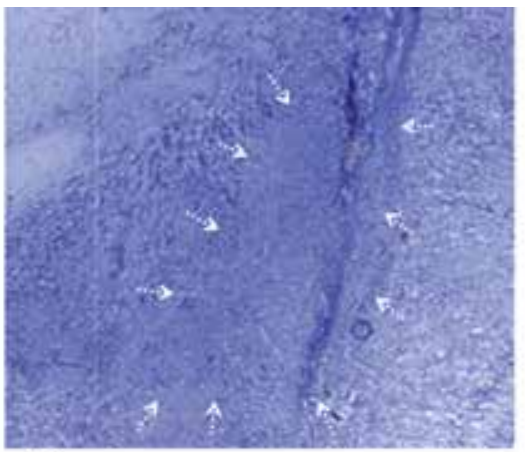

(B)

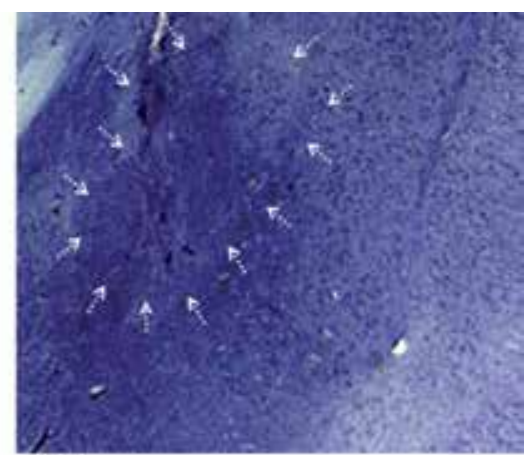

(C)

Figure 1. Histological results. Photomicrographs of Cresyl violet-stained coronal sections of the amygdala. (A) Representative sham lesion. (B) Representative lesion of the BLA. (C) Representative lesion of the CeA. Arrows denote lesion borders. Notice the sparse cells and gliosis, especially along the injector tracks in the lesioned areas. BLA, basolateral amygdala; CeA, central nucleus of the amygdala. 
Six BLA-lesioned brains were judged to have lesions including lesions of the lateral, basal, and accessory basal nuclei. These brains exhibited damage in the anterior portion of the BLA, and most rats also had damage to part of the posterior portion of the BLA. No lesions of the CeA were observed in any of the cases. Eight CeA-lesioned brains were judged to have acceptable lesions. These brains exhibited damage in the medial portion of the CeA. No cell loss was evident in any of the sham-operated brains.

Behavioral results. ANOVAs were used to analyze the average percentage of responses in the three groups (BLA, CeA, and sham-operated) of rats during the FI 60 -s schedule in the last three sessions of the prelesion training and revealed a significant effect only of period $\left(F_{3,64}=15060.002, p<.001\right)$. Newman-Keuls post hoc tests showed that the $0-15$-s and $16-30$-s periods were not significantly different from each other. The 31-45-s and 46-60-s periods were significantly different from each other and different from the other periods. A gradual increase in responding during the FI 60-s schedule was found, indicating that the rats in the three groups presented acquisition of FI 60 -s task performance.

Statistical analysis of post-lesion training showed that BLA and CeA lesions did not interfere with the maintenance of FI 60-s task performance. ANOVAs were used to analyze the average percentage of responses in the three groups of rats during the FI 60-s schedule in the three sessions of post-lesion training and revealed a significant effect only of period $\left(F_{3,64}=14664.321, p<\right.$ $.001)$. Newman-Keuls post hoc tests showed that all of the periods were significantly different from each other.

In the post-lesion testing (Figure 2), the average percentage of responses of the rats in the three groups during the $0-15$-s period after reinforcement (post-R) and after nonreinforcement (post-N) was analyzed. ANOVA did not show any effect of group $\left(F_{2,32}=8.049\right.$, $p=.65)$ but showed an effect of period $\left(F_{1,32}=1029.155\right.$, $p<.001)$. The average percentage of responses was higher in the beginning of the post-N intervals than in the beginning of the post- $\mathrm{R}$ intervals in the three groups

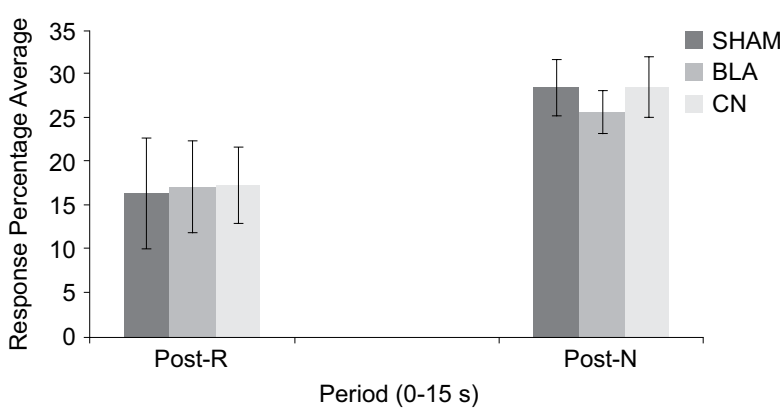

Figure 2. Post-lesion testing. Response percentage average during the first $15 \mathrm{~s}(0-15 \mathrm{~s}$ period) after reinforcement (post-R) and nonreinforcement (post-N) in the three sessions of post-lesion testing. BLA, group of rats with BLA lesions; $\mathrm{CeA}$, group of rats with CeA lesions; SHAM, group of shamoperated rats. of rats. These data indicate that BLA and CeA lesions did not interfere with the ROE.

\section{Discussion}

Although several studies showed that the amygdala may be involved in the modulation of attentional and motivational processes and, therefore, the modulation of processes that are likely involved in the ROE, the present results did not show that the BLA and CeA lesions interfered with the ROE. When the partial reinforcement schedule was introduced in the FI 60-s schedule procedure, rats in the three groups exhibited a higher response percentage average after nonreinforcement than after reinforcement (0-15-s period). Moreover, following nonreinforcement, no difference was found among the performance of the rats in the three groups.

These results are similar to the study of McDonough \&Manning(1979) where rats with lesions of the amygdala were compared with sham-operated rats in their response to the occasional omission of food following training on a FI 60 -s schedule. The results showed that amygdala lesions did not prevent the ROE. Rats with lesions of the amygdala were more responsive than rats in the shamoperated group following nonreinforcement. However, these findings conflict with Henke \& Maxwell (1973) and Henke (1977) who used runway paradigms. These data also do not support the results of Henke (1973) who used an operant conditioning paradigm. Because these studies induced amygdala lesions using radiofrequency (RF) or electrolytic current, elimination of the ROE that resulted from these techniques might be attributable to either extensive damage to the amygdala complex or destruction of fibers of passage (Salinas et al., 1996).

In the studies by Henke $(1973,1977)$, amygdala lesions were induced by RF or electrolytic current. The divergence between their findings and the present results of Experiment 1 do not appear to be related to the extent of the lesion. McDonough \& Manning (1979) also induced amygdala lesions using RF techniques, and their results did not show elimination of the ROE. Thus, effects of amygdala lesions on the ROE may be associated with the different procedures used (i.e., two components of an FI 120-s schedule in Henke (1973) and one component of a FI 60-s schedule in McDonough \& Manning (1979).

The results of Experiment 1 are also similar to those obtained by Salinas et al. (1996). These authors showed that none of the lesions, including lesions of the CeA and BLA and discrete lesions of the CeA, interfered with the negative contrast effect. However, Salinas et al. (1996) showed that discrete lesions of the BLA interfered with the negative contrast effect, but the present results showed that BLA lesions did not interfere with the ROE.

The lack of effect of amygdala lesions, especially BLA lesions, on the ROE in Experiment 1 is surprising. A large body of evidence suggests a role for the BLA in motivational processes. The BLA appears to play a more specific role in representing the affective value of conditioned stimuli. Such information may then be used 
to support the translation of conditioned associations into instrumental action (Whitelaw, Robbins, Everitt, \& Markou 1996; Parkinson, Robbins, \& Everitt, 2000).

\section{Experiment 2}

\section{Methods}

Subjects. Thirty two experimentally naive male Wistar rats, 90 days old at the beginning of the experiments and weighing 416-433 g were used in the study. Upon arrival, treatment of the rats was identical to Experiment 1.

Apparatus. The apparatus was similar to Experiment 1 , with the exception that the operant chambers were also equipped with a speaker that delivered a $1000-\mathrm{Hz}$, 30-dB tone (auditory stimulus).

Pre-lesion training. In the first session, each rat was placed in the experimental chamber and trained to lever press for water. In the second session, which lasted $30 \mathrm{~min}$, the animals were subjected to a CRF schedule in which lever pressing at any moment was always followed by reinforcement. The next day, the rats were trained on an FI 8-s schedule with a limited hold 2-s (LH 2 s) signaled schedule. The schedule was presented simultaneously with an auditory stimulus (pure tone) of $10 \mathrm{~s}$. The first lever press that occurred between 8 and $10 \mathrm{~s}$ resulted in water delivery $(100 \%$ reinforcement condition). All rats received 30 training trials per session. Each trial was interpolated with variable intertrial intervals (mean, $120 \mathrm{~s}$ ). Fifteen daily sessions of training were conducted. Throughout the experiment, training was conducted from Monday to Friday only. Each session lasted $50 \mathrm{~min}$. Rats were water-deprived for $\sim 23 \mathrm{~h}$ before the beginning of each session.

Surgical procedures. After pre-lesion training, the animals underwent stereotaxic surgery according to the atlas of Paxinos \& Watson (2007). Ten rats received bilateral lesions of the BLA. Eleven rats received bilateral lesions of the CeA. Eleven rats underwent sham surgery (sham-operated group). The surgical procedures were identical to Experiment 1.

Post-lesion training. After surgical recovery, rats were given 10 sessions of post-lesion training. These sessions were identical to the last sessions of the prelesion training (100\% reinforcement condition).

Post-lesion testing. After post-lesion training, 15 sessions in the FI 8-s LH 2-s signaled schedule were conducted, during which reinforcement omission was introduced in $23 \%$ of the trials (partial reinforcement condition). Reinforced and nonreinforced events were randomly distributed during the session using the criteria of up to three subsequent intervals with the same schedule.

Histology. Histological procedures were identical to Experiment 1.

Data analysis. For statistical analysis of the acquisition training on the FI 8-s LH 2-s signaled schedule, response averages during the FI 8-s in the pre- and post-lesion training were grouped into 4-s periods ( $1-4 \mathrm{~s} \mathrm{FI}$ and 5-8 $\mathrm{s}$ FI). For statistical analysis of the ROE in the post-lesion testing, the response averages during $6 \mathrm{~s}$ after the FI 8-s
LH 2-s signaled schedule were grouped into 2-s periods (2-, 4-, and 6-s). These data were analyzed using two-way ANOVA with group as the inter-group factor (BLA, CeA, and sham-operated groups) and period as the intra-group factor. ANOVA was followed by the Newman-Keuls post hoc test; $p \leq .05$ was accepted as statistically significant.

\section{Results}

Histological results. The reliability criteria for the lesions were identical to Experiment 1. Nine BLAlesioned brains were judged as having lesions, including lesions of the lateral, basal, and accessory basal nuclei. These brains exhibited damage in the anterior portion of the BLA, and most rats also had damage to part of the posterior portion of the BLA. No CeA lesion was found in any of the cases. Ten CeA-lesioned brains were judged as having acceptable lesions. These brains exhibited damage in the CeA medial portion. No cell loss was evident in any of the sham-operated brains.

Behavioral results. Statistical analysis of the pre-lesion training showed that all groups exhibited acquisition of the FI 8-s LH 2-s signaled schedule. ANOVAs were used to analyze the response averages in the three groups of rats during the FI 8-s periods in the last three sessions of the pre-lesion training and revealed only a significant effect of period $\left(F_{2,66}=14.83, p<.01\right)$. Newman-Keuls post hoc tests showed that the initial 4-s FI (1-4-s) was different from the final 4-s FI (5-8-s) in the three groups. An increase in responding was observed during the FI periods, indicating that performance was under temporal control. Thus, the three groups of rats exhibited acquisition of FI 8-s LH 2-s task performance.

Statistical analysis of the post-lesion training data showed that BLA and CeA lesions did not interfere with the acquisition of the FI 8-s LH 2-s signaled schedule. ANOVAs were used to analyze the average percentage of responses in the three groups of rats during the FI 8 -s periods in the three sessions of the post-lesion training and only revealed a significant effect of period $\left(F_{2,66}=5.001, p<.01\right)$. Newman-Keuls post hoc tests showed that the initial 4-s FI (1-4 s) was different from the final 4-s FI (5-8 s). An increase in responding was observed during the FI period. These data indicate that performance in the three groups of rats continued to be under the temporal control of the schedule.

In post-lesion testing (Figure 3), statistical analysis showed that the BLA and CeA lesions interfered with the ROE. The response averages in the three groups during the $6 \mathrm{~s}$ after reinforcement (post-R) and after the nonreinforcement (post-N) periods were analyzed. ANOVA revealed an effect of period $\left(F_{2,66}=3.45, p\right.$ $<.05$ ). Newman-Keuls post hoc tests showed that the response averages were higher at the beginning of the post-N periods (2-, 4-, and 6-s periods) than at the beginning of the post-R periods (2-, 4-, and 6-s periods) in the three groups, indicating a ROE. Furthermore, ANOVA revealed an effect of group after the post-N periods $\left(F_{2,110}=6.04, p=.03\right)$. However, Newman- 


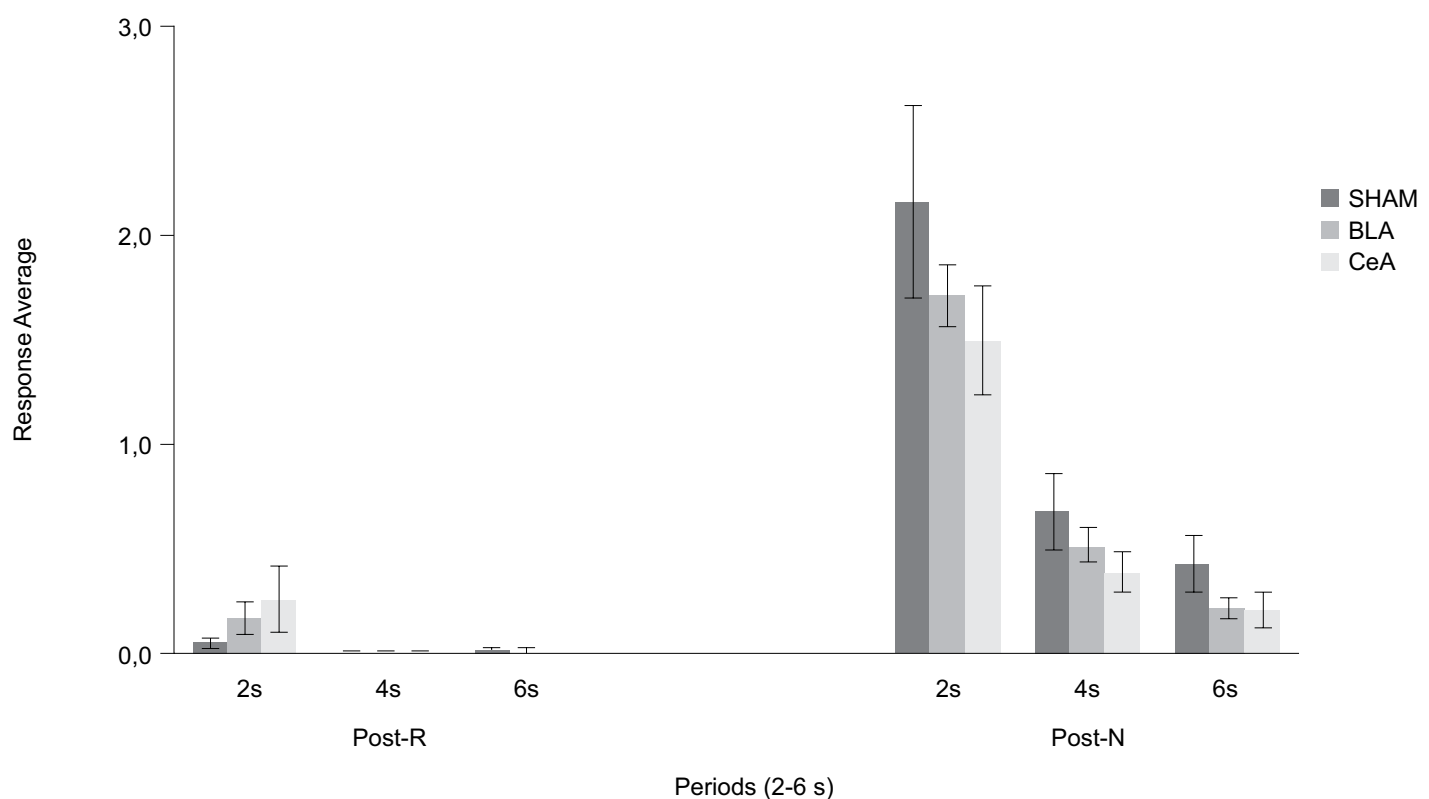

Figure 3. Post-lesion testing. Average lever presses during the $6 \mathrm{~s}$ after the FI $8 \mathrm{~s} \mathrm{LH} 2 \mathrm{~s}$ signaled schedule (2, 4, and $6 \mathrm{~s}$ periods) after reinforcement (Post-R) and nonreinforcement (Post-N) in the first sessions of post-lesion testing. BLA, group of rats with BLA lesions; CeA, group of rats with CeA lesions; SHAM, group of sham-operated rats.

Keuls post hoc tests did not reveal specific differences among the three groups in each post-N period $(2,4$, and $6 \mathrm{~s})$. BLA and CeA groups generally exhibited a ROE at a lower intensity than the control group.

\section{Discussion}

The results of post-lesion testing showed that BLA and CeA lesions interfered with the ROE. When the partial reinforcement schedule was introduced in the FI 8-s LH 2-s schedule procedure, rats in the three groups exhibited higher response averages after nonreinforcement than after reinforcement (2-, 4-, and 6-s periods). However, a difference was found between performance in the sham-operated group and both the CeA- and BLA-lesioned groups. The response rates in the lesioned groups were lower than in sham-operated group only in the post-N periods.

The present results are similar to Henke \& Maxwell (1973) and Henke (1977) who used runway paradigms, and Henke (1973) who used an operant conditioning paradigm (FI $120 \mathrm{~s}$ ). Although the data obtained in this experiment did not show that BLA and CeA lesions prevented the ROE, as in Henke's studies they suggest that lesions of the BLA and CeA reduce the ROE. Although McDonough \& Manning (1979) showed that rats with lesions of the amygdala that were trained to respond on a FI 60-s schedule were more responsive than rats in the sham-operated group following reinforcement omission, results of Experiment 2 in the present study showed the opposite.

The results of this experiment are also similar to Salinas et al. (1996), showing that discrete lesions of the BLA interfered with the negative contrast effect. Thus, motivational components related to BLA activation may be essential for the preservation of both the ROE and negative contrast effect. However, the present data do not support the findings that lesions of either the $\mathrm{CeA}$ or BLA and discrete lesions of the CeA did not interfere with the negative contrast effect.

The divergent results of studies investigating the role of the amygdala in the modulation of the ROE do not appear to be related to the techniques used to induce amygdala lesions. Thus, the training parameters or the extent of the amygdala lesions in each study may have influenced the results.

For example, Henke (1973) and McDonough \& Manning (1979) used an operant conditioning paradigm and omission procedure, which utilizes parameters similar to those in the present study. Henke (1973) and McDonough \& Manning (1979) used $50 \%$ of nonreinforced trials to evaluate the effect of amygdala lesions on the ROE, whereas the present experiment used 23\% of nonreinforced trials. Coughlin (1970) used different omission reinforcement percentages $(25 \%, 50 \%$, or $75 \%)$ in a double runway paradigm and showed that the magnitude of the ROE was an inverted U-shaped function of the percentage of reinforcement, with the $50 \%$ group showing the greatest ROE. Thus, the percentage of nonreinforced trials used to evaluate the ROE can also influence the results. Furthermore, in the Henke (1973) and McDonough \& Manning (1979) studies, the lesions extended to the entire amygdala, whereas the lesions were made in specific areas of the amygdala (i.e., CeA and BLA) in the present study.

\section{General discussion}

Some studies have suggested that the amygdala is involved in the modulation of responses that are 
correlated with motivational and attentional processes and in the modulation of processes that are likely involved in the ROE (Bueno et al., 2012). However, studies that have investigated the effect of extensive amygdala lesions on the ROE have presented divergent results. Henke (1973, 1977) and Henke \& Maxwell (1973) suggested that amygdala lesions prevented the ROE, but McDonough \& Manning (1979) showed that rats with amygdala lesions were more sensitive to reinforcement omission.

The present study examined the effects of selective amygdala lesions on the ROE and also did not obtain similar results. The results of Experiment 2-but not Experiment 1 - suggested that the amygdala is involved in the modulation of the ROE. In Experiment 1, rats in both groups with BLA and CeA lesions exhibited higher response rates after nonreinforcement than after reinforcement. No difference was found among the performance of three groups of rats following nonreinforcement. In Experiment 2, although rats in both groups with BLA and CeA lesions exhibited higher response rates after nonreinforcement than after reinforcement, these rates were lower than those of the sham-operated group. Thus, results of Experiment 2but not Experiment 1-suggest that the motivational and attention processes mediated by BLA and CeA activation are involved in the modulation of the ROE.

Differences between the procedures of Experiments 1 and 2 in the present study may have contributed to the different results. Although both experiments involved reinforcement omission procedures, some factors must be considered. In Experiment 1, the percentage of trials in which correct responses were not followed by reinforcement was $50 \%$. This percentage was $23 \%$ in Experiment 2. A lower percentage of trials followed by nonreinforcement may increase the surprising omission of reinforcement. Additionally, an exteroceptive signal was used in Experiment 2 but not in Experiment 1. According to Colwill \& Rescorla (1988), discriminative stimuli provide information about the reinforcer earned by a response in the normal course of instrumental learning. These predictions allow animals to evaluate future events before they actually occur and permit the selection and preparation of behavioral reactions. Thus, a discriminative stimulus induces an internal motivational state by evoking an expectation of the reinforcement (Schultz, 1998). The use of an exteroceptive signal in Experiment 2 may have enhanced reinforcement expectancy.

These general observations suggest that $\mathrm{CeA}$ and BLA lesions interfered with the ROE, depending on the training. The importance of training parameters in processes involved in the ROE was highlighted by Stout, Muzio, Boughner, \& Papini (2002) and Stout, Boughner, \& Papini (2003). The results of the present study suggest that the CeA and BLA may be involved in the modulation of the ROE when (1) the performance is under the control of exteroceptive signaling but not under temporal control, which is more automatic, and (2) the omission of reinforcement is more surprising.
The divergence between the results of Henke (1973, 1977) and Henke \& Maxwell (1973) and Experiment 1 in the present study does not appear to be related to the extent of the lesions. Henke (1973, 1977), Henke \& Maxwell (1973), and McDonough \& Manning (1979) induced extensive amygdala lesions using similar techniques and found opposite results. Thus, the effects of amygdala lesions on the ROE may be associated with the different procedures used. Although Henke (1973) and McDonough \& Manning (1979) used operant conditioning paradigms, the procedure used by Henke (1973) involved two components of a FI 120-s schedule, and the procedure used by McDonough \& Manning (1979) involved one component of a FI 60-s schedule.

The results of Experiment 2 and the findings presented by Judice-Daher et al. (2012) showed that selective lesions of the BLA and CeA did not eliminate, but rather interfered with, the ROE. However, in Experiment 2, CeA and BLA lesions reduced the ROE, whereas these lesions potentiated the ROE in the study of Judice-Daher et al. (2012). This divergence does not appear to be related to the behavioral procedures employed because in both cases a fixed-interval with limited hold signaled schedule was used. However, the surgical procedures used by Judice-Daher et al. (2012) were different from the present study. In the present study, the CeA lesions were designed to target a subregion of CeA connectivity that is thought to be critical for the expression of numerous attentional consequences of conditioning (Gonzales \& Chesselet, 1990; Gallagher \& Holland, 1992). These lesions spared substantial portions of the CeA, especially in more caudal regions that were reached in the study by Judice-Daher et al. (2012). Some studies have shown that these regions, because of their projections, are related to important motivational processes (Holland \& Gallagher, 2003).

Although recent evidence suggested a functional dissociation between different amygdala areas, data obtained in the present study did not show a doubledissociation between the CeA and BLA. In both experiments the performance of the rats with BLA lesions and the rats with $\mathrm{CeA}$ lesions after nonreinforcement was not different. The lack of differences between the lesioned groups in Experiments 1 and 2 did not allow the identification of dissociable attentional or motivational components of the ROE with functions of specific amygdala areas. Similar to the findings of Judice-Daher et al. (2012), these results did not show a functional double-dissociation between the $\mathrm{CeA}$ and BLA in the modulation of the ROE.

Two possibilities may be related to the absence of differences between the effects of CeA and BLA lesions on the ROE. Although recent evidence from appetitive conditioning studies suggests that the BLA and CeA operate in parallel to mediate distinct incentive processes, some studies that used aversive learning suggested that these areas function serially to mediate distinct aspects of emotional processing (Balleine \& 
Killcross, 2006). According to Papini \& Dudley (1997), the unexpected omission of reinforcement produces an aversive emotional reaction with physiological and behavioral consequences. Initially, the surprising omission of reinforcement induces an internal state with immediate consequences for the behavior of the organism, acting as aversive reinforcement (Papini, 2003). From this perspective, the BLA and CeA could operate serially to mediate the ROE.

The absence of specific effects of $\mathrm{CeA}$ and BLA lesions on the ROE may also be related to the complementarity of the motivational and attentional components involved in the ROE. According to Holland \& Gallagher (1999), despite such dissociations between specific amygdala subsystem functions, they must interact at some level. Motivation and emotion clearly play key roles in attention, and the direction of attention can have a substantial influence on emotional processing. Furthermore, anatomically, there are many opportunities for the convergence of information processed in these subsystems (Holland \& Gallagher, 1999). The amygdala has long been known to have many nuclei with several distinct functions and connections. These nuclei have a wide network both in neural interactions within the amygdala and in other areas of the brain. Identifying a single area responsible for a given function is not possible. The BLA is involved in the acquisition and representation of reinforcement value, apparently through its connections with ventral striatal dopamine systems and the orbitofrontal cortex. The CeA, however, contributes heavily to attentional and general motivational functions in conditioning through its influence on basal forebrain cholinergic systems and the dorsolateral striatum (Holland \& Gallagher, 1999; Cardinal, Parkinson, Hall, \& Everitt, 2003; Balleine \& Killcross, 2006).

\section{Acknowledgments}

J.L.O. Bueno received a research fellowship and research grants from the National Council for Technological and Scientific Development (CNPq, Brazil). D.M. Judice-Daher and T.F. Tavares received scholarships from Coordination for Improvement of Higher-Education Personnel (CAPES, Brazil) and postdoctoral fellowships from the São Paulo Research Foundation (FAPESP).

\section{References}

Amsel, A. (1962). Frustrative nonreward in partial reinforcement and discrimination learning: Some recent history and a theoretical extension. Psychological Review, 69(4), 306-328.

Amsel, A. (1992). Frustration theory: Many years later. Psychological Bulletin, 112(3), 396-399.

Amsel, A., \& Roussel, J. (1952). Motivational properties of frustration: I. Effect on a running response of the addition of frustration to the motivational complex. Journal of Experimental Psychology, 43, 363-368.

Balleine, B. W., \& Killcross, S. (2006). Parallel incentive processing: An integrated view of amygdala function. Trends in Neurosciences, 29(5), 272-279.

Balleine, B. W., Killcross, S., \& Dickinson, A. (2003). The effect of lesions of the basolateral amygdala on instrumental conditioning. Journal of Neuroscience, 23(2), 666-675.
Berlau, D. J., \& McGaugh, J. L. (2003). Basolateral amygdala lesions do not prevent memory of context-footshock training. Learning and Memory, 10, 495-502.

Blundell, P., Hall, G., \& Killcross, S. (2001). Lesions of the basolateral amygdala disrupt selective aspects of reinforcer representation in rats. Journal of Neuroscience, 21(22), 9018-9026.

Bueno, J. L., Judice-Daher, D. M., \& Tavares, T. F. (2012). Neurobiologia dos efeitos de expectativa e omissão de reforço sobre o comportamento. Avances em Psicologia Latinoamericana, in press.

Cardinal, R. N., Parkinson, J. A., Hall, J., \& Everitt, B. J. (2003). The contribution of the amygdala, nucleus accumbens, and prefrontal cortex to emotion and motivated behavior. International Congress Series, 1250, 347-370.

Colwill, R. M., \& Rescorla, R. A. (1988). Associations between the discriminative stimulus and the reinforcer in instrumental learning. Journal of Experimental Psychology: Animal Behavior Processes, 14(2), 155-164.

Coughlin, R. (1970). Frustration effect and resistance to extinction as a function of percentage of reinforcement. Journal of Experimental Psychology, 84(1), 113-119.

Everitt, B. J., Cardinal, R. N., Parkinson, J. A., \& Robbins, T. W. (2003). Appetitive behavior: Impact of amygdala-dependent mechanisms of emotional learning. Annals of the New York Academy of Sciences, 985, 233-250.

Gallagher, M., Graham, P. W., \& Holland. P. C. (1990). The amygdala central nucleus and appetitive Pavlovian conditioning: Lesions impair one class of conditioned behavior. Journal of Neuroscience, 10(6), 1906-1911.

Gallagher, M., \& Holland, P. C. (1992). Understanding the function of the central nucleus: Is simple conditioning enough? In J. P. Aggleton (Ed.), The amygdala: neurobiological aspects of emotion, memory, and mental dysfunction (pp. 307-321). New York: Wiley-Liss.

Gonzales, C., \& Chesselet, M. F. (1990). Amygdalonigral pathway: An anterograde study in the rat with Phaseolus vulgaris leucoagglutinin (PHA-L). Journal of Comparative Neurology, 297, 182-200.

Hatfield, T., Han, J. S., Conley, M., Gallagher, M., \& Holland, P. (1996). Neurotoxic lesions of basolateral, but not central, amygdala interfere with Pavlovian second-order conditioning and reinforcer devaluation effects. Journal of Neuroscience, 16(16), 5256-5265.

Henke, P. G. (1973). Effects of reinforcement omission on rats with lesion in the amygdala. Journal of Comparative and Physiological Psychology, 84(1), 187-193.

Henke, P. G. (1977). Dissociation of the frustration effect and the partial reinforcement extinction effect after limbic lesions in rats. Journal of Comparative and Physiological Psychology, 91(5), 1032-1038.

Henke, P. G., \& Maxwell, D. (1973). Lesions in the amygdala and the frustration effect. Physiology and Behavior, 10, 647-650.

Holland, P. C., \& Gallagher, M. (1993a). Amygdala central nucleus lesions disrupt increments, but not decrements, in conditioned stimulus processing. Behavioral Neuroscience, 107(2), 246-253.

Holland, P. C., \& Gallagher, M. (1993b). Effects of amygdala central nucleus lesions on blocking and unblocking. Behavioral Neuroscience, 107(2), 235-245.

Holland, P. C., \& Gallagher, M. (1999). Amygdala circuitry in attentional and representational processes. Trends in Cognitive Sciences, 3(2), 65-73.

Holland, P. C., \& Gallagher, M. (2003). Double dissociation of the effects of lesions of basolateral and central amygdala on conditioned stimulus-potentiated feeding and Pavlovian-instrumental transfer. European Journal of Neuroscience, 17, 1680-1694.

Judice-Daher, D. M., Tavares, T. F., \& Bueno, J. L. O. (2011). Influence of the reinforcement magnitude on omission effects. Behavioural Processes, 88(1), 60-62.

Judice-Daher, D. M., Tavares, T. F., \& Bueno, J. L. O. (2012). Involvement of the basolateral complex and central nucleus of amygdala in the omission effects of different magnitudes of reinforcement. Behavioural Brain Research, 233, 149-156.

McDonough, J. H., \& Manning, F. J. (1979). The effects of lesions in amygdala or dorsomedial frontal cortex on reinforcement omission and noncontingent reinforcement in rats. Physiological Psychology, 7, 167-172.

Papini, M. R. (2003). Comparative psychology of surprising nonreward. Brain, Behavior and Evolution, 62, 83-95.

Papini, M. R., \& Dudley, R. T. (1997). Consequences of surprising reward omissions. Review of General Psychology, 1, 175-197.

Parkinson, J. A., Robbins, T. W., \& Everitt, B. J. (2000). Dissociable roles of the central and basolateral amygdala in appetitive emotional learning. European Journal of Neuroscience, 12(1), 405-413. 
Paxinos, G., \& Watson, C. (2007). The rat brain in stereotaxic coordinates, 6th edition. San Diego: Academic Press.

Salinas, J. A., Parent, M. B., \& McGaugh, J. L. (1996). Ibotenic acid lesions of the amygdala basolateral complex or central nucleus differentially effect the response to reductions in reward. Brain Research, 742, 283-293.

Salinas, J. A., \& White, N. M. (1998). Contributions of the hippocampus, amygdala, and dorsal striatum to the response elicited by reward reduction. Behavioral Neuroscience, 112(4), 812-826.

Schultz, W. (1998). Predictive reward signal of dopamine neurons. Journal of Neurophysiology, 80, 1-27.

Seward, J. P., Pereboom, A. C., Butler, B., \& Jones, R. B. (1957). The role of prefeeding in an apparent frustration effect. Journal of Experimental Psychology, 54, 445-450.
Staddon, J. E. R. (1974). Temporal control, attention, and memory. Psychological Review, 81(5), 375-391.

Stout, S. C., Boughner, R. L., \& Papini, M. R. (2003). Reexamining the frustration effect in rats: Aftereffects of surprising reinforcement and nonreinforcement. Learning and Motivation, 34, 437-456.

Stout, S. C., Muzio, R. N., Boughner, R. L., \& Papini, M. R. (2002). Aftereffects of the surprising presentation and omission of appetitive reinforcers on keypecking performance in pigeons. Journal of Experimental Psychology: Animal Behavior Processes, $28,242-256$.

Whitelaw, R. B., Robbins, T. W., Everitt, B. J., \& Markou, A. (1996). Excitotoxic lesions of the basolateral amygdala impair the acquisition of cocaine-seeking behaviour under a second-order schedule of reinforcement. Psychopharmacology, 127(3), 213-224. 
